\title{
EL ENTE COMARCAL EN LA LEY CATALANA DE ALTA MONTAÑA
}

352.071: 353.094 .4 (46. Cataluña)

por

Joaquín Tornos Mas

SUMARIO: I. LA POLITICA DE MONTAÑA EN EL MARCO DE LA CONSTITUCION. LA LEY ESTATAL DE AGRICULTURA DE MONTAÑA.-II. LA LEY CATALANA DE ALTA MONTAÑA: NOTAS GENERALES: 1. LA DELIMITACIÓN DE LAS AREAS DE ACTUACIÓN. 2. El Plan COMO TÉCNica de actuactón. 3. Los Consejos COMARCALES.-III. LOS CONSEJOS COMARCALES DE MONTANa: 1. NaTURaleza JURfDica. 2. ORganización.-IV. LA FIGURA DE LOS CONSEJOS COMARCALES EN EL PROCESO DE REORGANIZACION TERRITORIAL.-V. ANEXO: NORMATIVA DE LA GENERALIDAD DE CATALUÑA RELATIVA A LOS CONSEJOS COMARCALES DE MONTAÑA.

I. LA POLITICA DE MONTAÑA EN EL MARCO DE LA CONSTITUCION. LA LEY ESTATAL DE AGRICULTURA DE MONTAÑA

La reflexión que vamos a realizar en las páginas siguientes en torno al fenómeno comarcal y su tratamiento jurídico en la Ley catalana de Alta Montaña, debe enmarcarse en la política de montaña a que alude el artículo 130, 2, de la Constitución Española. 
No cabe duda que la preocupación por dar respuesta a una grave situación de desequilibrios territoriales claramente localizados en las zonas de montaña, así como las experiencias extranjeras desarrolladas en este tema y la propia presión de las colectividades implicadas, llevó al constituyente a incluir en la Constitución un mandato expreso dirigido a todos los poderes públicos para que dispensaran un tratamiento especial a las zonas de montaña. Los poderes públicos quedaban así emplazados para adoptar las soluciones más adecuadas en orden a frenar el proceso de degradación de las zonas de montaña y conseguir su equiparación con otras zonas del país. Obviamente, y la propia ubicación del artículo 130 en el Título VII de la Constitución así lo indica, la política de montaña tiene un contenido claramente económico. Si tuviéramos que analizar en profundidad este tema, deberíamos prestar especial atención a las medidas jurídicas que dan cobertura e impulsan las acciones tendentes a conseguir el desarrollo de estas zonas. Nuestro interés, sin embargo, se centra en otro aspecto propio de la política de montaña, en concreto, la implicación territorial y organizativa de la citada política, que al plantearse como objetivo el desarrollo de unas áreas determinadas de la geografía española adquiere una inevitable vertiente organizativa o de delimitación de espacios afectados.

Es evidente que si se pretende dar un tratamiento especial a unas zonas determinadas, el primer objetivo de la Ley consistirá en establecer cuáles son estas áreas territoriales. A partir de este momento puede pensarse en la creación de meras circunscripciones territoriales en las que aplicar la política de un Ente territorial (o de varios, a través de la elaboración de un Plan con intervención de los sujetos implicados), o bien en dotar de cierta entidad jurídica a la zona de montaña, reconociendo la existencia de unos intereses propios que precisan un Ente exponencial de la Comunidad afectada, o un Ente de gestión para la prestación de ciertos servicios.

Situados en esta perspectiva, cobra todo su sentido la Ley catalana de 9 de marzo de 1983, en tanto, como hemos de ver, la Comarca de montaña recibe un trato jurídico especial a través de la creación y personificación de los Consejos comarcales de montaña. Otra solución diversa es la ofrecida por la Ley estatal de Agricultura de Montaña (Ley 25/1982, de 30 de junio), sobre cuyo contenido nos detendremos ahora brevemente. 
La citada Ley, como señala su artículo primero, tiene como objeto el «establecimiento de un régimen jurídico especial para las zonas de agricultura de montaña con el fin de posibilitar su desarrollo social y económico, especialmente en sus aspectos agrarios, manteniendo un nivel demográfico adecuado y atendiendo a la conservación y restauración del medio físico, como hábitat de sus problemas».

El objetivo es, como se ve, establecer un régimen jurídico especial para unas zonas, con el fin de posibilitar su desarrollo. En consecuencia, primero deben delimitarse las zonas, y en segundo lugar, utilizar los instrumentos que la Ley facilita. Para ello, la Ley va a establecer dos tipos de disposiciones: por una parte, la fijación de las áreas de actuación, y por otra, la introducción del programa de ordenación como principal técnica de actuación.

En relación con el primer punto, la Ley estatal opta de forma clara por la simple delimitación de unas circunscripciones en las que aplicar una política. En el aspecto organizativo no aparece, pues, ningún nuevo Ente al que se atribuyan funciones propias para definir los intereses de la zona o gestionarlos.

Los artículos segundo y tercero determinan qué debe entenderse por zonas de agricultura de montaña o áreas de alta montaña, vinculando la obtención de los beneficios de la Ley a la calificación expresa de constituir uno $u$ otro tipo de circunscripción territorial (artículo cuarto). Los criterios para llegar a constituir una zona de agricultura de montaña o área de alta montaña son puramente fácticos (altura, vocación agraria, tipo de vegetación), pudiendo integrarse en estas circunscripciones "Comarcas, términos municipales o partes de los mismos» (art. segundo, párr. primero).

En relación con la articulación de la política de montaña, la Ley introduce la técnica de los programas de ordenación y promoción, en cuya elaboración participarán «todas las Entidades territoriales afectadas, debiendo ser oídas, en períodos de información pública, las Asociaciones de montaña y personas interesadas" (artículo décimo). En este sentido, debe destacarse la referencia a las Asociaciones de montaña, en tanto se trata de una figura también introducida por la Ley (arts. quince a diecisiete) como mecanismo de participación de sujetos interesados en los temas de agricultura de montaña. Así, junto a los Entes territoriales y Entidades representativas 
de intereses profesionales o económicos, se regulan e impulsan Asociaciones civiles que canalicen los intereses de sujetos afectados para el mejor desarrollo de los objetivos previstos en la Ley.

Las citadas Asociaciones participarán en la elaboración del Programa de ordenación y en la redacción de las Ordenanzas para el uso de las zonas de agricultura de montaña. La Asociación podrá, por tanto, recoger y expresar aquellos intereses que se identifican de forma más directa con la problemática propia del área de montaña.

En definitiva, la política de montaña se ha traducido, en el aspecto organizativo, en la delimitación de unas áreas de actuación y en la regulación de unas Asociaciones de montaña cuya constitución y actuación se deja al impulso de los afectados (obviamos en este momento los problemas relativos a la competencia para aprobar las delimitaciones de las áreas de actuación, cuestión que la disposición adicional de la Ley estatal, con la remisión a su artículo quinto, resuelve a favor del Gobierno, en base a una discutible cobertura del artículo 149, 1-23, de la Constitución. El apartado a) del citado artículo quinto ha sido impugnado por el Gobierno Vasco ante el Tribunal Constitucional, publicándose en el $B O E$ de 27 de octubre de 1982 la admisión a trámite del recurso).

En el aspecto funcional $u$ operativo, el instrumento central es el Programa de ordenación, cuya aprobación corresponde al Gobierno y cuya ejecución se remite a las diversas Administraciones públicas en virtud de sus respectivas competencias (arts. quinto, apartado b), y once).

\section{LA LEY CATALANA DE ALTA MONTAÑA: NOTAS GENERALES}

La Ley del Parlamento catalán de 9 de marzo de 1983, de Alta Montaña, parte de una preocupación coincidente con la que impulsó la Ley estatal a que acabamos de referirnos. La exposición de motivos del texto del Parlamento catalán es extraordinariamente significativa en este sentido, con referencias expresas al artículo 130, 2, de la Constitución, a la legislación europea, a las directivas del Consejo de Europa. En el fondo subyace el mismo interés por articular una política de montaña, si bien ahora enunciada en su 
alcance general y no reducida al tema agrícola (debe, no obstante, reconocerse en este punto que el contenido de la Ley estatal desborda su estrecho enunciado).

La respuesta a esta preocupación común va a orientarse por caminos también semejantes, haciendo pivotar el entramado legal sobre dos grandes ejes: el Plan comarcal de montaña y los Consejos comarcales, instituciones a través de las cuales se trata de conseguir el desarrollo de las Comarcas de montaña. Como puede observarse, las cuestiones organizativas han sufrido un cambio sustancial con relación a los planteamientos de la Ley de Agricultura de Montaña. La Comarca de montaña es el área territorial de actuación principal, y como tal se define y delimita de forma precisa en la Ley. Por otra parte, se crean unos Consejos comarcales como Entidades públicas de naturaleza local, Entes de participación claramente diferenciados de las Asociaciones de montaña. Estos aspectos organizativos, y en particular la figura de los Consejos comarcales, van a ocupar nuestra atención principal al examinar la Ley catalana.

\section{LA DELIMitación DE LAS AREAS DE ACTUACION}

La Ley de Alta Montaña determina, en su artículo primero, que su objetivo es establecer "un régimen jurídico específico para las Comarcas y zonas de montaña", con la finalidad de alcanzar algunos logros concretos, que pasan a enumerarse a continuación. Se contiene, pues, una referencia concreta a dos áreas de actuación, que se definen en los artículos segundo y tercero (también reciben un trato especial las áreas de montaña que se encuentran en cotas superiores al límite natural del bosque autóctono de la zona, artículo cuarto, párrafo sexto).

De las dos áreas citadas merece especial atención la Comarca de montaña, pues en torno a la misma gira toda la dinámica de la Ley (la zona de montaña es una solución a problemas de menor extensión y sólo da lugar a un trato de favor en ayudas y subvenciones; vid. el art. noveno). Además, la utilización del término Comarca suscita de inmediato la cuestión de si se está introduciendo el Ente comarcal como primer paso de un proceso más amplio de reordenación territorial. Pues bien, en este sentido, deben destacarse las siguientes cuestiones: 
1. $\quad$ La Comarca se utiliza como demarcación territorial sobre la que aplicar el Plan comarcal, pero no se personifica como Ente de ninguna clase.

2. A lo largo de todo el proceso de elaboración de la Ley estuvo presente la implicación del contenido de la misma con el tema de la organización territorial, quedando patente en las intervenciones de los Diputados que no se quería abordar el tema de la organización territorial (vid. el Diario de Sesiones del Parlamento Catalán, 1983, Serie P, núm. 121, fasc. 1 y 2). Igualmente, la referencia que en un determinado momento contenía el Proyecto de Ley al artículo quinto, párrafo tercero, del Estatuto catalán desapareció en la redacción definitiva.

3. Las referencias que en algún artículo de la Ley se hace a la Comarca como si se tratara de un Ente con personalidad propia, deben entenderse como imperfecciones técnicas, pues, como se ha dicho, en ningún momento la Comarca es configurada como Ente. Así, la referencia a la "coordinación de las Comarcas» que se contiene en el artículo decimotercero, párrafo segundo, apartado $c$ ), o la imposición al Consell Executiu de la Generalidad de dotar a las Comarcas de los bienes y medios para cumplir sus funciones, que se dispone en la transitoria segunda, deben entenderse como referencias a los Consejos comarcales.

La Comarca, pues, es simple circunscripción territorial que delimita la aplicación de una política de desarrollo contenida en el Plan comarcal.

Admitido esto, debe igualmente destacarse que la Ley catalana delimita de forma concreta este ámbito territorial, más allá, pues, de la fijación de unas características generales que podrían dar lugar a la fijación de una zona de actuación en una fase posterior (como ocurre en la Ley estatal de Agricultura de Montaña). Así, y tras señalar en el artículo segundo, párrafo primero, las notas que definen la Comarca de montaña, en el párrafo segundo enumera qué Comarcas se consideran Comarcas de montaña. Este hecho vuelve de nuevo a introducir el tema del Ente comarcal y la organización territorial. Si son Comarcas de montaña unas determinadas Comarcas, ello supone que la institución comarcal existe como división territorial. Sobra, por tanto, una definición de las características 
que definen la Comarca de montaña, y basta con señalar qué Comarcas son de montaña.

Esta segunda opción es la que en verdad adopta la Ley catalana, para lo cual, y ante la falta de una definición legal de la Comarca en el sistema jurídico vigente, se recurre al antecedente histórico de la Comarca republicana. Así, la disposición adicional de la Ley determina que el ámbito territorial de las Comarcas de montaña, establecidas en el artículo 2, 2, se entiende que hace referencia al que fue aprobado por los Decretos del Gobierno de la Generalidad de los días 27 de agosto y 23 de diciembre de 1936 (demarcación comarcal que en su día fue simple administración periférica de la Generalidad, todo ello dentro de un período de guerra).

Pero, en todo caso, se trata de una simple técnica para la fijación de meras circunscripciones territoriales sobre las cuales llevar a la práctica un Plan comarcal de actuación. No surge, pues, tampoco desde esta perspectiva, Ente alguno, si bien la relación con el tema de la ordenación territorial está subyacente. Así, la propia Ley incluye una disposición transitoria que revela esa mala conciencia del legislador por acercarse demasiado a un tema que no era el propio de una Ley de montaña. La disposición transitoria primera señala que: «los artículos y disposiciones de la Ley relativos a las Comarcas de montaña serán válidos mientras la Ley de División Territorial de Cataluña, de que habla el artículo quinto, párrafo tercero, del Estatuto, y la legislación que en materia de Régimen local elabore el Parlamento en uso de la competencia atribuida por el artículo noveno, párrafo octavo, del Estatuto, no dispongan lo contrario". Es decir, cuando se organice territorialmente la Generalidad y se creen los Entes territoriales, la Comarca de montaña, mera circunscripción territorial, deberá adecuar su extensión al nuevo Ente comarcal, desapareciendo tanto en su extensión como en su naturaleza actual.

Las conexiones temáticas con la organización territorial de Cataluña y las, a nuestro juicio, incorrecciones de la Ley catalana, no deben llevar, sin embargo, a pensar que la Ley de Alta Montaña ha creado el Ente comarcal. La Ley catalana es una Ley relativa a la política de montaña y, a tal fin, delimita unas zonas para aplicar esta política, recurriendo, eso sí, al antecedente histórico de la circunscripción comarcal republicana (referencia a la Comarca re- 
publicana que aparece también en la Ley del Parlamento Catalán de Política Territorial de 21 de noviembre de 1983, en su disposición transitoria).

Puede pensarse en una clara vocación para recoger el fenómeno comarcal y recuperar unos ámbitos de cierta raíz histórica, pero insistimos, la Comarca no se crea como Ente de ningún tipo.

\section{El Plan como técnica de actuación}

La Ley de Alta Montaña deposita toda su confianza en el Plan comarcal de montaña, regulado en sus líneas generales en los artículos cuarto a séptimo de la Ley. El citado Plan, acompañado de un Programa de actuación, se configura como el instrumento capaz de coordinar las actuaciones de las distintas Administraciones en el área comarcal estableciendo el necesario orden de prioridades entre aquéllas. Desde el punto de vista competencial y procedimental, se trata de un Plan de la Generalidad de Cataluña (la aprobación definitiva y su aplicación corresponde al Consell Executiu), fruto de un proceso de elaboración en el que se intenta conseguir la más amplia participación de los sujetos afectados. Los aspectos relativos a la gestión quedan en un segundo plano, siendo, sin duda, una de las cuestiones que precisa de inmediato un mayor desarrollo y clarificación (en general, sobre estos temas concernientes a los aspectos jurídicos del Plan, me remito a la comunicación presentada en estas mismas Jornadas por el profesor FoNT LlOVET; téngase también en cuenta el Decreto 84/1984, de 16 de marzo, de desarrollo de la Ley de Alta Montaña, en el que se abordan algunas de las cuestiones aquí mencionadas; DOG núm. 429 de 1984).

\section{Los Consejos comarcales}

Regulados en el artículo 15 de la Ley constituyen la aportación más novedosa de là citada norma, en tanto se personifica el órgano de gobierno y no, como es habitual, el Ente conformado por un territorio, una población y una organización determinada. No se personifica la Comarca, sino el Consejo comarcal, como si en lugar de personificar el Municipio o la Provincia, se personificase el Ayuntamiento o la Diputación. 
La importancia del tema requiere un tratamiento especial, que abordamos en el epígrafe siguiente.

\section{LOS CONSEJOS COMARCALES DE MONTAÑA}

Vamos a realizar el estudio de los Consejos comarcales de montaña atendiendo, en primer lugar, a su naturaleza jurídica, para después prestar atención a su organización.

\section{Naturaleza jurídica}

El artículo decimoquinto de la Ley determina que en cada Comarca deberá constituirse un Consejo comarcal que tendrá plena personalidad jurídica, naturaleza territorial y carácter representativo. Este Consejo personificado surge como fruto de una enmienda de última hora presentada por el Diputado Luna SERrano, el cual la justificó en los siguientes términos: "lo que buscamos es una estructuración institucional, de manera que se tienda a precisar los rasgos definitorios de estos Consejos comarcales dotándoles de plena capacidad y personalidad jurídica, indicando su naturaleza territorial por cuanto que actúan sobre la Comarca e indicando, además, sut carácter representativo por cuanto que han de llevar a cabo un cometido de carácter público en beneficio de la Comarca y tienen que ser expresión de las propias fuerzas sociales y políticas comarcales» (Diario de Sesiones, cit., pág. 3646). Frente a la personificación de la Comarca, en último momento, se dota de personalidad' jurídica lo que el Proyecto de Ley calificaba como simple órgano. de participación.

Estamos, sin duda, ante una figura atípica cuya naturaleza debe ser precisada a partir de un examen del conjunto del texto legal. En este sentido, es de gran interés atender a las funciones encomendadas al Consejo comarcal. El citado artículo 15, en su apartado segundo, atribuye al Consejo comarcal las funciones de representar y defender los intereses generales de la Comarca de montaña, solicitar la elaboración y revisión del Plan, informar del proyecto del Plan y aprobarlo inicial y provisionalmente, informar los programas de carácter intercomarcal, elaborar los Estatutos y normas organizativas internas y cualquier otra que por Ley se le atribuya. Por su 
parte, el artículo 12 determina que corresponde al Consell Executiu de la Generalidad la aprobación definitiva de los Planes comarcales de montaña, así como su ejecución, a cuyo efecto incluirá en el proyecto de presupuesto las previsiones financieras contenidas en los diversos planes comarcales (art. octavo).

¿Qué significan estos datos? A nuestro modo de ver, podemos concluir que el Consejo comarcal no supone la creación de un nuevo Ente territorial en sentido estricto, sino la aparición de un Ente de participación, exponencial de los intereses generales de una determinada comunidad, en concreto la comunidad comarcal.

Como ya apuntamos al tratar el tema de la Comarca como mera circunscripción territorial, la Ley de Alta Montaña no ha creado una nueva figura de Administración territorial. Ni era la intención del legislador ni es algo que pueda deducirse de un análisis de la voluntad de la Ley.

Tampoco se ha pretendido introducir un Ente institucional, orientado exclusivamente hacia fines de gestión, prestacionales, buscando una mayor racionalidad en la actuación administrativa.

El Consejo comarcal adquiere así el carácter de ser el Ente exponencial de una colectividad, vinculada por unos problemas comunes, lo que permite obtener una representación de los intereses generales de la misma. Este Ente representa y defiende estos intereses generales, para lo cual se arbitra un mecanismo de especial relevancia: la elaboración participada del Plan de montaña. Frente a una participación sectorial o corporativa, frente al impulso de unas Asociaciones en forma privada (recordar las Asociaciones de la Ley estatal de Agricultura de Montaña), se crean unos Consejos basados en la representación municipal y, por tanto, exponentes de intereses generales.

Esta misma idea subyace en la experiencia italiana. Las «Comunita Montane» se definen en su Ley de creación como Entes de participación en la programación. Así, el artículo primero de la Ley de 3 de diciembre de 1971 establece que «las disposiciones de la presente Ley están destinadas, de acuerdo con el artículo 44, apartado último, y 129 de la Constitución, a promover el desarrollo de las zonas de montaña, favoreciendo la participación de la población a través de las Comarcas de montaña, la preparación y gestión de los programas de desarrollo y del Plan territorial del respectivo 
compresorio de montaña, a fin de alcanzar una política general de reequilibrio económico y social en el marco de las indicaciones del programa económico y nacional y de los programas regionales». Esta naturaleza de Ente de participación en la programación se ha recogido con mayor fuerza en los Estatutos de alguna «Comunita Montane». Así, en los Estatutos de las «Comunita» del Alto Lacio Occidental o en la del Apenino Regiano. En este último, se define la «Comunita» como «unidad territorial de programación democrática».

El Consejo comarcal de montaña no es, pues, un Ente territorial. Si se hubiera pretendido introducir un Ente de esta naturaleza, se hubiera personificado la Comarca, y como consecuencia hubiera surgido un Ente con autonomía para la gestión de los intereses de la comunidad incluida o comprendida en este ámbito territorial. También, como lógica derivación de este proceso, el Ente territorial habría gozado de las potestades inherentes a este tipo de Entes (normativa, tributaria, expropiatoria, etc.), potestades precisas para la satisfacción de los intereses de la comunidad a la que sirve con el carácter de administración territorial. Frente a esta posibilidad, rechazada expresamente por los Diputados (con la oposición de los grupos parlamentarios del PSC-PSOE y PSUC, que defendieron la personificación de la Comarca y la creación de una verdadero Ente territorial), se personifica el Consejo, que representa los intereses de los Municipios incluidos en el área comarcal y, por tanto, los intereses generales de la población de la Comarca. Como Ente representativo y de participación, se le atribuyen las funciones a que antes hicimos referencia, funciones no de gestión, que no permiten la satisfacción directa de las necesidades de la comunidad comarcal.

\section{ORGANIZACIÓN}

La Ley de Alta Montaña remite la organización de los Consejos comarcales a la potestad organizativa de los propios Consejos, al reconocer como una función de los mismos la «elaboración de sus Estatutos y de sus normas de funcionamiento" (art. quince, apartado segundo, letra $e$ )).

La Ley, no obstante, establece algunas reglas imperativas de carácter general. Así, determina que los Consejos estarán integrados 
por dos representantes de cada uno de los Municipios pertenecientes a su ámbito territorial, elegidos por el Ayuntamiento respectivo, uno de los cuales, por lo menos, debe ser Concejal municipal (artículo quince, apartado primero). Igualmente determina cuál debe ser el aparato administrativo mínimo de que deben disponer los Consejos comarcales y cuáles serán las normas que regirán su funcionamiento.

La previsión legal, en cuanto a los Estatutos y composición de los Consejos, ha sido desarrollada por sendos Decretos del Gobierno de la Generalidad.

a) El Decreto 297/1983, de 14 de julio (DOG núm. 347, de 22 de julio), establece el procedimiento para la elección de los miembros de los Consejos comarcales, elección que la Ley atribuía a cada Ayuntamiento. Como datos a destàcar, en este proceso electoral, pueden señalarse los siguientes:

- Los candidatos se proponen por un número mínimo preestablecido de Concejales en proporción al número de miembros del Ayuntamiento.

- Se aplican supletoriamente las disposiciones de la Ley de Elecciones locales de 17 de julio de 1978.

- Se confeccionan dos listas de candidatos. Una con aquellos que, a su vez, sean Concejales, y otra con todos los candidatos.

- Primero se votará la lista de los Concejales-candidatos, y a continuación la lista de todos los candidatos, excluyéndose los que ya hubieran resultado elegidos en la primera votación. De esta forma se garantiza que, al menos, uno de los representantes del Municipio sea Concejal, pudiendo el otro resultar elegido entre personas que no forman parte del Consistorio municipal.

- Las votaciones se resolverán con el voto favorable de la mayoría simple, prevista en el artículo segundo de la Ley 40/1981, de 28 de octubre.

- Las votaciones se celebran en sesión plenaria de cada Ayuntamiento, convocada expresamente con esta finalidad, que será la única en el orden del día.

- La duración del mandato de los elegidos es de cuatro años.

- En caso de inactividad por parte de algún Municipio, el Conseller de Governació, a solicitud del Consejo comarcal o de un nú- 
mero de vecinos afectados no inferior al 20 por 100 , podrá nombrar a los dos representantes del Municipio afectado.

A la vista de estas disposiciones relativas a la elección del Consejo comarcal pueden efectuarse algunas objeciones críticas.

La elección del Consejo a partir de los Municipios que se integran en la Comarca, estableciendo para cada Municipio dos representantes, crea un Consejo comarcal desvinculado de la población comarcal y estrechamente ligado a los Entes municipales. La elección de segundo grado debería haber mantenido cierta proporción con la población de los Entes municipales y, por tanto, con el principio de un hombre, un voto.

Por otra parte, tampoco se garantiza que la composición del Consejo respete el pluralismo político que pueda darse dentro de cada uno de los Municipios que forman parte del Consejo comarcal. Cada Municipio elige sus dos representantes en una doble votación sobre listas distintas por mayoría simple, de manera que cualquier grupo con minoría-mayoritaria puede colocar a sus dos candidatos en el Consejo. Ello puede llevar a la creación de un Ente representativo conformado por un único partido, cuando a nivel de los respectivos Municipios existan diversas opciones políticas que no se ven reflejadas en el Consejo.

Si el Consejo comarcal es Ente de representación debería haberse cuidado más la proporcionalidad con la representación política de los Municipios, y a través suyo con las opciones políticas de la comunidad comarcal.

Por último, no creemos que tenga excesiva justificación la introducción de personas que no pertenezcan a los Consistorios municipales, tratándose de una elección de segundo grado realizada por los propios Concejales. La incorporación de técnicos o expertos al Consejo puede arbitrarse por otras vías, manteniendo, en cambio, la relación directa entre Municipio y Consejo comarcal a través de esta identidad personal de los Concejales municipales y los miembros del Consejo comarcal.

b) Si bien la Ley de Alta Montaña remite la elaboración de los Estatutos a la decisión del Consejo comarcal respectivo, el Decreto citado en el epígrafe anterior, en su disposición transitoria segunda, reconoció al Consejo ejecutivo de la Generalidad la potestad de fijar unos «criterios básicos para la elaboración de los Estatutos, respe- 
tando las potestades de autoorganización de los Consejos». Es en base a esta discutible habilitación, en tanto que reduce la amplia remisión a la potestad estatutaria contenida en la Ley de Alta Montaña (art. quince, ap. segundo, letra $e$ )), que se dictó el Decreto de 6 de octubre de 1983, por el cual se fijaron unos criterios básicos para la elaboración de los Estatutos de cada Consejo.

Junto a unas mínimas normas de tipo procedimental, se determina el contenido de los Estatutos y se impone una organizacióntipo basada en tres órganos: presidente-órgano plenario-órgano ejecutivo, a los que se atribuyen unas funciones determinadas. Poco queda, pues, al Consejo comarcal, que deberá traducir lo dicho en el modelo impuesto a la situación concreta y llenar los escasos huecos que deja el Decreto de la Generalidad. Mayor libertad se reconoce en cuanto a las normas de funcionamiento y en lo concerniente al Presupuesto, si bien en estos temas la vinculación se impone a través de la remisión a la legislación local.

En resumen, pues, la elaboración de los Estatutos pone de relieve una fuerte injerencia de la Administración de la Generalidad de Cataluña, que asume un papel de Ente coordinador de los Consejos comarcales en el momento de su puesta en funcionamiento. Toda la política de montaña, en la que se integran los Consejos, es una política de la Generalidad. Si el Plan, instrumento básico, corresponde a esta última en su aprobación y gestión, el Ente de participación que la Ley catalana crea no podía quedar desvinculado de la Administración de la Generalidad, lo que explica su intervención en estos aspectos estatutarios. Es la Administración de la Generalidad la que busca un interlocutor válido para elaborar su Plan, y por ello dispone cómo debe configurarse, nombrando incluso sus miembros si los Municipios no actúan (disposiciones adicionales segunda y tercera del Decreto 279/1983, de 14 de julio).

El análisis de la naturaleza y organización de los Consejos comarcales permite concluir, por tanto, que nos encontramos con un Ente atípico, que en ningún caso puede equipararse al Ente comarcal, si bien responde también en última instancia al fenómeno comarcal como realidad indiscutible dentro de la vida social y política catalana, fenómeno especialmente sentido en las áreas de montaña.

Esta figura de los Consejos comarcales de montaña puede verse como una solución únicamente válida para la política de montaña, como respuesta con un simple valor transitorio ante el proceso de 
reordenación territorial y, por tanto, llamada a desaparecer, o como experiencia de interés dentro de la futura reorganización territorial, superando su especialización para las áreas de montaña y convirtiéndose no en una técnica coyuntural y limitada territorialmente, sino en una técnica a generalizar en todos los sentidos. La convicción de que esta última opción es posible nos ha llevado a prestar especial atención a esta figura de los Consejos comarcales, opción que tratamos de justificar en el próximo epígrafe.

\section{LA FIGURA DE LOS CONSEJOS COMARCALES EN EL PROCESO DE REORGANIZACION TERRITORIAL}

El proceso de reorganización territorial, que a través de la potestad compartida Estado-Comunidades Autónomas, va a ponerse en marcha tras la aprobación de la Ley de Bases de Régimen local, puede encontrar en la experiencia de los Consejos comarcales de montaña un interesante punto de reflexión.

No cabe duda que la temática comarcal va a estar presente en el debate ligado al proceso de reorganización territorial, y de forma particular en algunas Comunidades Autónomas. En este sentido, y teniendo en cuenta que la Constitución garantiza la autonomía de Municipios y Provincias (idea reforzada en el Proyecto de Ley de Bases del Régimen local), ¿cabe seguir pensando en la Comarca como Ente territorial? ¿Es preciso un nuevo Ente intermedio de estas características? La respuesta a estas cuestiones no debería partir de presupuestos históricos, ni de exclusivas interpretaciones jurídicas de los textos en vigor. La respuesta debe plantearse no a través de la defensa o crítica de unas instituciones a las que se alude de forma abstracta, sino planteando la situación de los administrados y buscando aquellas instituciones que en cada momento sean más eficaces y respondan mejor a sus intereses.

Desde esta perspectiva entendemos quee deben mantenerse únicamente tres niveles de Administración territorial: Comunidad Autónoma, Provincia y Municipio, sin perjuicio de redefinir los ámbitos territoriales de Provincias y Municipios a través de las técnicas jurídicas precisas en cada caso.

En este nuevo modelo territorial, la Comarca puede pasar a desempeñar un papel importante como Administración institucional, 
reconociéndosele la gestión de algunos concretos servicios municipales, en particular los de carácter supramunicipal. No obstante, la Ley de Alta Montaña ofrece otra vía posible para reconocer el fenómeno comarcal que supone la ventaja de introducir un Ente de participación al lado de una nueva técnica de actuación: la planificación. En lugar de buscar la solución a los problemas del pequeño Municipio y del área comarcal en la creación de una figura organizativa, se acude a una técnica funcional. Al mismo tiempo, para desarrollar el principio de la democracia, el pluralismo y la participación, se crea un Ente de participación que democratiza el Plan, lo vincula a los intereses de la zona y ofrece a los Municipios un cauce para conseguir su intervención en aquellos asuntos de su interés que desborden su capacidad de actuación. El fenómeno comarcal se concreta así a través de la creación de los Consejos comarcales.

Sin duda, el gran reto que introduce esta nueva técnica es el relativo a la gestión de este Plan participado, pues de la eficacia de esta gestión depende la valoración positiva o negativa de todo el sistema. Para ello pueden mantenerse las Administraciones existentes, si bien coordinadas por un Plan que resuelva los problemas que alcanzan un nivel comarcal, Plan que, por otra parte, estas mismas Administraciones han elaborado (por ello, si bien el Plan debe aprobarse por la Comunidad Autónoma, como Ente superior, debería contar con la participación de todos los Entes que quedan vinculados en su ejecución, y entre ellos, la Provincia).

En definitiva, si el Plan comarcal deja de ser el Plan de la Generalidad para una zona de su territorio, y se convierte en el Plan de las Entidades territoriales afectadas por la ordenación del ámbito comarcal, podemos estar ante una solución alternativa a la creación de un nuevo Ente territorial o institucional. La representación de la «voluntad» comarcal se conseguiría a través del Ente de «participación», es decir, el Consejo comarcial.

De esta forma se consigue:

1. Se configura un Ente que representa y define los intereses supramunicipales con una especial interrelación, es decir, los intereses que están en la base del sentimiento comarcal. La necesidad de reconocer tales intereses no supone, por tanto, el reconocimiento de su definición y actuación a un Ente superior. 
2. El Plan o Programa es el resultado de una elaboración participada y democrática a través de un Ente en el que se resumen de forma coordinada los intereses generales representados por cada Municipio. Esta participación convertirá al Plan en un instrumento de mayor eficacia en su aplicación, en tanto será asumido como propio por los Entes en cuyo territorio vaya a aplicarse. El Consejo comarcal, por otra parte, podrá efectuar una labor de fiscalización directa sobre la aplicación del Plan.

3. No se crea un nuevo Ente territorial, de difícil encaje entre la Provincia y el Municipio, y por contra se obtiene un mecanismo operativo para dar respuesta a las necesidades de las áreas comarcales, contando con un Ente representativo que expresa cuáles son estas necesidades.

4. El Consejo comarcal puede convertirse, por último, en una fórmula de potenciación de la autonomía municipal, entendiendo la misma no como el ejercicio de competencias separadas, sino como la participación en todos aquellos asuntos que inciden en la esfera de intereses municipales.

\section{ANEXO: NORMATIVA DE LA GENERALIDAD DE CATALUÑA RELATIVA A LOS CONSEJOS COMARCALES DE MONTAÑA}

Ley de Alta Montaña. Ley 2/1983, de 9 de marzo. DOG número 312 de 16 de marzo.

Decreto $297 / 1983$, de 14 de julio, por el que se establece el procedimiento para la elección de los miembros de los Consejos comarcales de montaña y su constitución (DOG núm. 347 de 22 de julio).

Decreto 414/1983, de 6 de octubre, por el que se establecen los criterios básicos para la elaboración de los Estatutos de los Consejos comarcales de montaña (DOG núm. 372 de 14 de octubre).

Decreto 426/1983, de 18 de octubre, por el que se otorgan a los Consejos comarcales de montaña las subvenciones previstas (DOG número 377 de 2 de noviembre).

Decreto 566/1983, de 7 de diciembre. Desarrollo reglamentario de la Ley 2/1983, de 9 de marzo, de Alta Montaña. Establece las fun- 
ciones de la Dirección General de Política Territorial (DOG núm. 400 de 20 de enero).

Decreto 84/1984, de 16 de marzo. Desarrollo de la Ley de Alta Montaña. Desarrolla los preceptos relativos al Consejo General de Montaña y a los Planes comarcales de montaña. 